\title{
Revista Colombiana de

\section{Evaluación de las características morfológicas de la aurícula izquierda por tomografía computarizada multicorte}

\author{
Juan C. Díaz ${ }^{a, *}$, Juan C. Uribe ${ }^{b}$, Pedro Abad-Díaz ${ }^{c}$, Mauricio Duque ${ }^{d}$, \\ Julián M. Aristizábal ${ }^{d}$, Jorge E. Velásquez ${ }^{d}$, Jorge E. Marín ${ }^{d}$, \\ Jorge A. Delgado ${ }^{c}$, Laura Duque ${ }^{a}$ y William Uribe ${ }^{d}$
}

\author{
a Universidad CES, Medellín, Colombia \\ b Clínica Las Vegas, Medellín, Colombia \\ c Fundación Instituto de Alta Tecnología Médica de Antioquia, Medellín, Colombia \\ d Cardiología y Electrofisiología, CES Cardiología, Medellín, Colombia
}

Recibido el 15 de octubre de 2014; aceptado el 6 de mayo de 2015

Disponible en Internet el 19 de agosto de 2015

\author{
PALABRAS CLAVE \\ Fibrilación auricular; \\ Aurícula izquierda; \\ Tomografía; \\ Venas; \\ Ablación atrial
}

\begin{abstract}
Resumen
Objetivo: Describir las características morfológicas de la aurícula izquierda, las venas pulmonares y la orejuela mediante tomografía computarizada en pacientes con fibrilación auricular paroxística o persistente, así como su posible impacto en el aislamiento eléctrico de venas pulmonares y la exclusión percutánea de la orejuela izquierda.

Métodos: Se revisaron las imágenes obtenidas mediante tomografía computarizada de todos los pacientes mayores de 18 años con fibrilación auricular paroxística o persistente, sometidos a ablación de venas pulmonares entre el 10. de enero de 2011 y el 30 de junio de 2012. Se excluyeron aquellos con antecedentes de ablación previa de venas pulmonares, fibrilación auricular permanente y cirugía cardíaca previa. Se hizo reconstrucción multiplanar, reconstrucción tridimensional de la anatomía y reconstrucción endoluminal en casos seleccionados.

Resultados: Se incluyeron 46 pacientes (37 hombres; 80,5\%), con un promedio de edad de $59,7 \pm 11,1$ años (rango 22 a 81 años). Se encontró alta prevalencia de venas accesorias, en su mayoría derechas, y un solo caso de venas accesorias izquierdas. Así mismo, se halló mayor diámetro del ostium de las venas pulmonares en comparación con lo descrito previamente, y mayor distancia a la primera bifurcación. De 46 pacientes, $11(24 \%)$ tuvieron un diámetro de la orejuela mayor de $28,5 \mathrm{~mm}$ y $9(19,5 \%)$ un diámetro que superaba los $30 \mathrm{~mm}$, puntos de corte establecidos para el uso de dispositivo de cierre de la orejuela.
\end{abstract}

\footnotetext{
* Autor para correspondencia.

Correo electrónico: jcdiaz1234@gmail.com (J.C. Díaz).
} 
Conclusión: Existen diferencias poblacionales en el drenaje venoso pulmonar y en la anatomía, que deben tenerse en cuenta al realizar intervenciones para el manejo de la fibrilación auricular. (c) 2015 Sociedad Colombiana de Cardiología y Cirugía Cardiovascular. Publicado por Elsevier España, S.L.U. Este es un artículo Open Access bajo la licencia CC BY-NC-ND (http://creativecommons.org/licenses/by-nc-nd/4.0/).

\section{KEYWORDS \\ Fibrillation; \\ Left atrium; \\ Tomography; \\ Veins; \\ Ablation}

\section{Determination of the anatomical characteristics of the left atrium by computed tomography}

\begin{abstract}
Objective: Describe the anatomical characteristics of the left atrium and pulmonary veins in patients with paroxysmal or persistent atrial fibrillation using computed tomography images and the possible impact these might have on pulmonary vein ablation and percutaneous left atrial appendage occlusion.

Methods: Evaluation of computed tomography images from adult patients with paroxysmal or persistent atrial fibrillation who were treated with pulmonary vein ablation from January $1^{\text {st }}$, 2011 to June $30^{\text {th }}$ 2012. Patients with previous cardiac surgery, pulmonary vein ablation or permanent atrial fibrillation were excluded. Multiplanar reconstrucion was performed in all patients, with three dimensional and endoluminal reconstruction performed in select patients. Results: Forty six patients (37 male, $80.5 \%$ ) with a mean age of $59.7 \pm 11.1$ years (range: $22-81$ years) were included. Accessory veins were common, most of them on the right side with only one case of left sided accessory vein. A larger size of the venous ostia and a longer distance from the ostia to the first bifurcation than previously described was found. A total of 11 patients (24\%) had a left atrial appendage ostium diameter larger than $28.5 \mathrm{~mm}$ and 9 patients (19.5\%) had a diameter larger than $30 \mathrm{~mm}$, which is the maximum limit for the use of left atrial appendage devices.

Conclusions: Anatomical characteristics of the left atrium and pulmonary veins have variations in different populations which should be taken into account prior to pulmonary vein ablation and left atrial appendage occlusion.

(C) 2015 Sociedad Colombiana de Cardiología y Cirugía Cardiovascular. Published by Elsevier España, S.L.U. This is an open access article under the CC BY-NC-ND license (http://creativecommons.org/licenses/by-nc-nd/4.0/).
\end{abstract}

\section{Introducción}

En el mundo, la fibrilación auricular es la arritmia sostenida más prevalente en la población general, con una alta tasa de morbimortalidad asociada a fenómenos embólicos (aumenta en cinco veces el riesgo de embolia cerebral), deterioro de la clase funcional y consultas al servicio de urgencias ${ }^{1,2}$. Todo lo anterior la convierte en una enfermedad de alto costo, característica que ha llevado a la búsqueda de estrategias para disminuir su impacto en la salud de los individuos y su costo para la sociedad. Por tanto, surgen estrategias de manejo médico (control de la respuesta ventricular, control del ritmo, anticoagulación) y técnicas invasivas, como el aislamiento eléctrico de las venas pulmonares para el tratamiento de la arritmia y de manera más reciente el cierre percutáneo de la orejuela auricular izquierda (OAI), con el objetivo de disminuir los fenómenos embólicos sin el uso de anticoagulantes, ambos procedimientos efectuados de manera cada vez más frecuente en las últimas dos décadas.

El aislamiento eléctrico de las venas pulmonares se fundamenta en la presencia de fibras musculares (denominadas «mangas» musculares), que se extienden desde la aurícula izquierda hacia el interior de las venas pulmonares, situación que se explica por su origen embriológico común, las cuales son responsables de iniciar hasta el $94 \%$ de los episodios de fibrilación auricular paroxística ${ }^{3}$. Este procedimiento, descrito inicialmente en 1998 por Haissaguerre et al. ${ }^{3,}$ y la técnica desarrollada más tarde por múltiples autores, han revolucionado el manejo de la fibrilación auricular al permitir modificar por primera vez el sustrato arritmogénico con la restauración subsiguiente del ritmo sinusal sin necesidad de usar antiarrítmicos en pacientes con fibrilación auricular paroxística o persistente. De manera paralela, en la última década ha surgido un interés en el uso de dispositivos percutáneos para el cierre de la OAI como parte de una estrategia que busca disminuir el riesgo embólico en pacientes con fibrilación auricular sin requerimiento del uso de anticoagulantes a largo plazo ${ }^{4}$. Esta iniciativa se fundamenta en que en pacientes con fibrilación auricular no valvular, aproximadamente el $90 \%$ de los trombos se originan en la orejuela ${ }^{5-7}$. Con el fin de lograr un acoplamiento adecuado del dispositivo con la orejuela izquierda, se requiere conocer las características morfológicas de la misma.

A ello se suma que hasta un $40 \%$ de los pacientes pueden tener variantes anatómicas de la aurícula izquierda o de las venas pulmonares, algunas de las cuales se pueden correlacionar con mayor dificultad en la realización de la ablación o del implante de un dispositivo de cierre percutáneo de la $\mathrm{OAl}^{8,9}$. Se sabe que las dimensiones de la aurícula izquierda y las venas pulmonares varían de acuerdo con la duración 
de la fibrilación auricular, siendo de mayor tamaño en aquellos pacientes que tienen fibrilación auricular persistente en comparación con aquellos con fibrilación auricular paroxística, y estos a su vez tienen mayores dimensiones que los pacientes en ritmo sinusal ${ }^{8,10,11}$. Lo mismo sucede con la anatomía de las venas pulmonares, las cuales al parecer poseen variaciones anatómicas más comúnmente en pacientes con fibrilación auricular que en pacientes en ritmo sinusal, fenómeno que sugiere un sustrato anatómico importante en la génesis y el mantenimiento de la arritmia, el cual progresa a medida que la enfermedad también lo hace ${ }^{11,12}$. Por último, la tasa de complicaciones (particularmente la de estenosis de las venas pulmonares posterior a la ablación), está en estrecha relación no solo con el lugar donde se realiza la ablación (mayor con la ablación ostial en comparación con la antral), sino que incluso es más alta cuando la distancia entre el ostium y la primera ramificación de la vena pulmonar es menor a $1 \mathrm{~cm}^{13}$. Algo similar sucede con el riesgo de fístula atrioesofágica, ya que el conocimiento de la posición del esófago respecto a la de las venas pulmonares y la pared posterior (y la presencia o no de grasa interpuesta entre venas pulmonares y el esófago), puede ser de ayuda para disminuir el riesgo de lesiones esofágicas ${ }^{14}$.

Por estas razones, el conocimiento previo de la morfología de la aurícula izquierda en el uso de técnicas como la angiografía rotacional, la tomografía computarizada multicorte, la resonancia magnética y la ecografía intracardíaca es fundamental, ya que permite una planeación adecuada, y mejora así la tasa de éxito y de posibles complicaciones. Dado que algunas de las características pueden variar de una población a otra (particularmente los índices de tamaño y de volumen), es importante determinar las características morfológicas de la aurícula izquierda en nuestra población. Este estudio se diseñó con base en este hecho.

\section{Métodos}

Se incluyeron todos los pacientes mayores de 18 años con fibrilación auricular paroxística o persistente, sometidos a aislamiento eléctrico de las venas pulmonares entre el $1 . \circ$. de enero de 2011 y el 30 de junio de 2012 en las Clínicas CES, Las Américas y Las Vegas, de Medellín, Colombia, a quienes se les realizó tomografía computarizada como parte del protocolo previo al procedimiento, la cual se analizó con el fin de determinar las características morfológicas de la aurícula izquierda. Se excluyeron pacientes con antecedente de ablación previa de venas pulmonares, fibrilación auricular permanente y cirugía cardíaca.

\section{Protocolo de la tomografía}

Los pacientes cumplieron con un ayuno mínimo de 6 horas para la realización de las tomografías, las cuales se hicieron con un equipo multidetector de 64 cortes (Phillips Brilliance, Phillips Medical Systems ${ }^{\circledR}$ ), utilizando medio de contraste de alta densidad (370 mg de yodo no iónico; Ultravist, Bayer ${ }^{\circledR}$ ) en una dosis de 1-2 $\mathrm{ml}$ por $\mathrm{kg}$ de peso con infusión de alta velocidad (4,5-5,5 ml por minuto), en inyector dual con un «bolus chase» de solución salina. Para la adquisición de imágenes se utilizó un protocolo de gatillado retrospectivo en fase diastólica, cuando el ritmo y la respuesta ventricular del paciente lo permitieron (en caso de fibrilación auricular con respuesta ventricular mayor a 100 lpm se efectuó sin gatillado). Para optimizar la adquisición de las imágenes se aplicó la técnica de «bolus tracking», utilizando un umbral entre 110 y 130 unidades Hounsfield (UH) en la aurícula izquierda con el objetivo de alcanzar una atenuación intraauricular mayor de $300 \mathrm{UH}$.

\section{Análisis de las imágenes y determinación de las características morfológicas}

Esta etapa se llevó a cabo en una estación de trabajo Extended Brilliance Workspace versión, 4.0.2.145 (Phillips Healthcare ${ }^{\circledR}$ ). Se hizo reconstrucción multiplanar, «tridimensional volumen rendering», así como navegaciones endoluminales en casos seleccionados.

\section{Características morfológicas de la aurícula izquierda}

El volumen de la aurícula izquierda se calculó utilizando la segmentación automatizada con exclusión de los ostium de las venas pulmonares y de la apertura de la OAl. La morfología de esta se determinó mediante su visualización en reconstrucción tridimensional, a través de la clasificación establecida por Koplay et al. ${ }^{15}$.

- Tipo 1: punta del apéndice orientada de manera superior, paralela a la arteria pulmonar.

- Tipo 2a: apéndice larga (con forma de dedo) que se orienta de manera inferior, paralela a la arteria pulmonar.

- Tipo 2 b: apéndice corta y poco prominente; se orienta de manera inferior paralela a la arteria pulmonar.

- Tipo 2c: apéndice ancha con orientación inferior de manera paralela a la arteria pulmonar, dándole forma de ala.

- Tipo 3: apéndice que inicialmente tiene una orientación superior pero que luego toma una dirección medial, ubicándose entre la arteria pulmonar y el cuerpo de la aurícula izquierda.

- Tipo 4: apéndice que inicialmente tiene una orientación superior pero que luego toma una dirección posterior.

- Tipo 5: apéndice que inicialmente tiene una orientación superior pero que posteriormente toma una dirección anterior.

Se eligió esta clasificación dada la diferenciación que hace de las orejuelas en distintos subtipos, con base no solo en la forma sino en la dirección que toman, característica importante para algunas intervenciones que podrían utilizarse en el futuro para el cierre de orejuela (i. e.: uso de dispositivo LARIAT $^{\circledR}$ ). El tamaño del ostium de la OAI se determinó mediante reconstrucción multiplanar con un corte axial a nivel del mismo, obteniendo mediciones de su diámetro mayor y menor. La longitud de la OAl se estableció con la herramienta de medición en curva, utilizando la reconstrucción multiplanar.

Para la evaluación del istmo mitral se usó la reconstrucción multiplanar, ubicando una proyección de cuatro cámaras. Se definió el istmo mitral como el área ubicada entre la inserción de la valva anterior de la válvula mitral y 


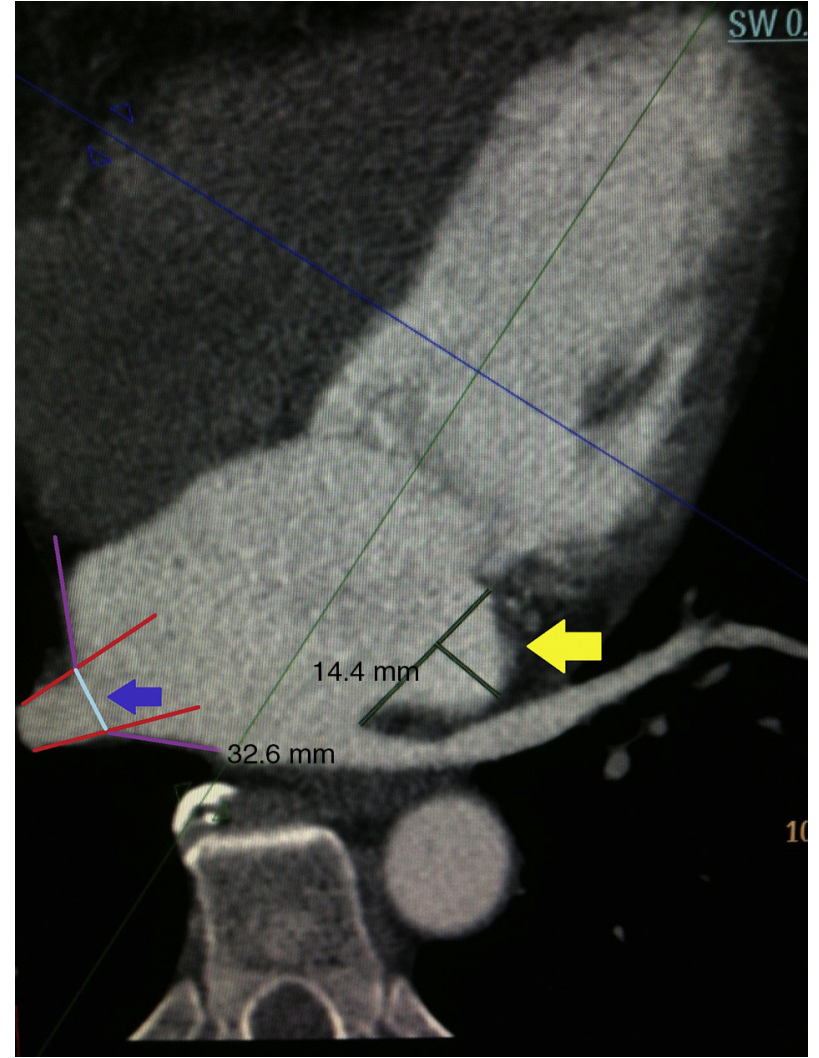

Figura 1 Flecha amarilla: medición del istmo mitral. Utilizando una reconstrucción ortogonal similar a la imagen de 4 cámaras cardíacas, se ubicó el istmo mitral localizado entre la inserción de la valva anterior de la válvula mitral y el ostium de la vena pulmonar inferior izquierda. La medición de la longitud se realizó trazando una línea recta entre ambos puntos. La profundidad del istmo se determinó trazando una línea perpendicular desde esa línea inicial hasta el borde de la aurícula y se consideró una evaginación del istmo mitral cuando esta superaba los $10 \mathrm{~mm}$. En este caso, la longitud del istmo es de $32,6 \mathrm{~mm}$ y la profundidad de $14,4 \mathrm{~mm}$. Flecha azul: determinación del ostium de la vena pulmonar. Se definió como ostium el punto de intersección entre la línea trazada por la pared de la aurícula (línea morada) y la línea trazada por la pared de la vena pulmonar (línea roja). El color de esta figura solo puede apreciarse en la versión electrónica del artículo.

el ostium de la vena pulmonar inferior izquierda. La medición de la longitud se realizó trazando una línea recta entre estos dos puntos (fig. 1). De acuerdo con lo que se ha descrito previamente, se consideró una evaginación del istmo mitral cuando la profundidad de este (medida como la distancia entre la línea antes descrita y el punto más profundo del istmo mitral) superaba los $10 \mathrm{~mm}^{16}$.

Se revisaron las paredes auriculares en los cortes axiales a fin de evaluar la presencia de divertículos (definidos como evaginaciones de la pared auricular).

\section{Características de las venas pulmonares}

El número de venas pulmonares y la presencia de venas accesorias se determinó en las imágenes de reconstrucción
Tabla 1 Características generales de la población y anatómicas de la aurícula izquierda

\begin{tabular}{ll}
\hline Variable & Resultado \\
\hline Edad promedio (años) & $59,7 \pm 11,1$ \\
Sexo masculino & $37(80,5 \%)$ \\
Volumen de la aurícula & $110,4 \pm 49,1 \mathrm{~cm}^{3}$ \\
Características de la orejuela & \\
$\quad$ Longitud & $44 \pm 12,2 \mathrm{~mm}$ \\
$\quad$ Ostium (diámetro mayor) & $26,3 \pm 5,1 \mathrm{~mm}$ \\
$\quad$ Ostium (diámetro menor) & $18,4 \pm 3,9 \mathrm{~mm}$ \\
Presencia de divertículo & $5(11 \%)$ \\
Evaginación del istmo mitral & 16 pacientes (35\%) \\
Longitud del istmo mitral & $31,8 \pm 8,3 \mathrm{~mm}$ \\
Contacto con el esófago & $28(60,8)$ \\
$\quad$ Grasa interpuesta & $40,5 \%$ \\
\hline
\end{tabular}

tridimensional. Se definió el ostium de la vena pulmonar como el punto de intersección entre la línea trazada a lo largo de la pared de la vena pulmonar y la línea trazada a lo largo de la pared de la aurícula izquierda (fig. 1); se consideró un ostium común cuando la totalidad o una parte de la circunferencia de este era compartida entre dos venas pulmonares. A través de reconstrucción multiplanar se determinó la distancia entre el ostium de la vena pulmonar y el punto de origen de la primera bifurcación, haciendo una medición lineal. Adicionalmente, se estableció el diámetro mayor y menor de la vena pulmonar a través de medición lineal.

\section{Relación de la aurícula con el esófago}

La relación del esófago con las venas pulmonares se evaluó en los cortes axiales, determinando si se encontraban en contacto, en cuyo caso se establecía a qué distancia estaban y si había o no grasa interpuesta.

\section{Análisis estadístico}

Los resultados se analizaron con el software SPSS statistics v. 21 (IBM software). Las variables cuantitativas se presentan con medidas de tendencia central como el promedio y de dispersión como la desviación estándar. Las variables categóricas se presentaron como proporciones.

\section{Resultados}

Se recogieron datos de 46 pacientes (37 hombres; 80,5\%) que cumplieron con los criterios de inclusión, con un promedio de edad de 59,7 $\pm 11,1$ años (rango 22 a 81 años). Las características de los pacientes y la anatomía de la aurícula izquierda se muestran en la tabla 1.

\section{Características morfológicas de la aurícula izquierda}

El volumen promedio de la aurícula izquierda fue de $110,4 \pm 49,1 \mathrm{~cm}^{3}$ (rango: 56-293 ml). Cinco pacientes (11\%) 


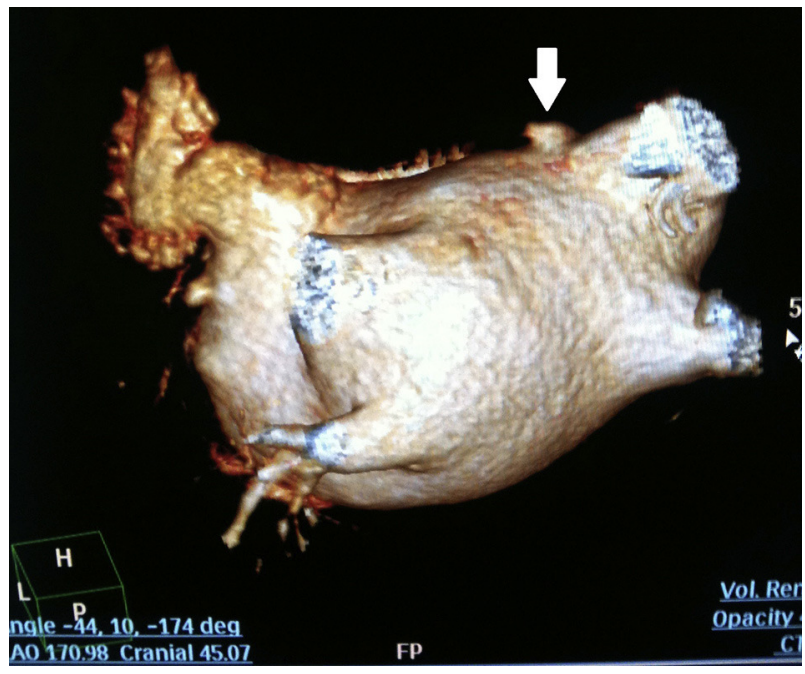

Figura 2 Divertículo auricular (flecha blanca).

tuvieron divertículos auriculares, todos ellos en la pared anterior de la aurícula (fig. 2) y $16(35 \%)$ tuvieron evaginaciones del istmo mitral, cuya profundidad promedio fue de $12,9 \pm 2,1 \mathrm{~mm}$ (fig. 3). La longitud del istmo mitral fue de $31,8 \pm 8,3 \mathrm{~mm}$.

Desde el punto de vista morfológico, el tipo más común de OAl fue el 2 a (14 pacientes, 30,4\%), seguido por el 1 (9 pacientes, $19,5 \%)$, el $2 \mathrm{~b}$ ( 9 pacientes, $19,5 \%)$, el $2 \mathrm{c}(7$ pacientes, $15,2 \%)$, el tipo 3 (3 pacientes, $6,5 \%)$ y finalmente el 4 y 5 (un paciente cada uno, 2,1\%). En dos casos se encontraron morfologías que no se ajustaron a la clasificación establecida. La longitud promedio de la OAI fue de $44 \pm 12,2 \mathrm{~mm}$, con unas dimensiones del ostium de $26,3 \pm 5,1 \mathrm{~mm}$ en su diámetro mayor y de $18,4 \pm 3,9 \mathrm{~mm}$ en su diámetro menor.

\section{Características de las venas pulmonares}

Las características morfológicas de las venas pulmonares se resumen en la tabla 2 . Se documentaron venas pulmonares accesorias en 12 pacientes (26\%), la mayoría (11 pacientes) en el lado derecho. Solo un paciente tenía más de una vena accesoria (dos venas accesorias en el lado derecho), en tanto que 6 (13\%) tenían ostium común izquierdo; en ninguno se encontró ostium común derecho.

En la mayoría de los casos, los ostium tuvieron una morfología ovoide, con un diámetro mayor claramente definido. Esta diferencia entre el diámetro mayor y el menor fue más evidente en las venas del lado izquierdo.

La menor distancia a la primera bifurcación se encontró en las venas inferiores derechas (promedio $8,7 \pm 5,1 \mathrm{~mm}$ ), mientras que la mayor distancia la tenían las venas superiores izquierdas $(22,7 \pm 10,4 \mathrm{~mm})$.

\section{Relación de las venas pulmonares y la aurícula con el esófago}

En 28 pacientes $(60,8 \%)$ las venas pulmonares se encontraban en contacto con el esófago; en todos los casos el contacto fue con las venas izquierdas excepto en un paciente en quien además del contacto con estas se observó contacto con la vena inferior derecha. En las venas izquierdas, el contacto con la vena inferior se presentó en 18 pacientes y con la vena superior en 23 (en 12 hubo contacto con la vena superior y con la vena inferior izquierda). La distancia promedio a la vena superior izquierda fue de $2,9 \mathrm{~mm}$ y de $1,7 \mathrm{~mm}$ a la vena inferior izquierda. En el paciente que se encontró contacto del esófago con la vena inferior derecha, la distancia fue de $1,1 \mathrm{~mm}$.

Sumando todas las venas en contacto, se encontraron 42 contactos vena-esófago. En tan solo 17 (40,5\%) de estos contactos se halló grasa interpuesta; en los demás casos
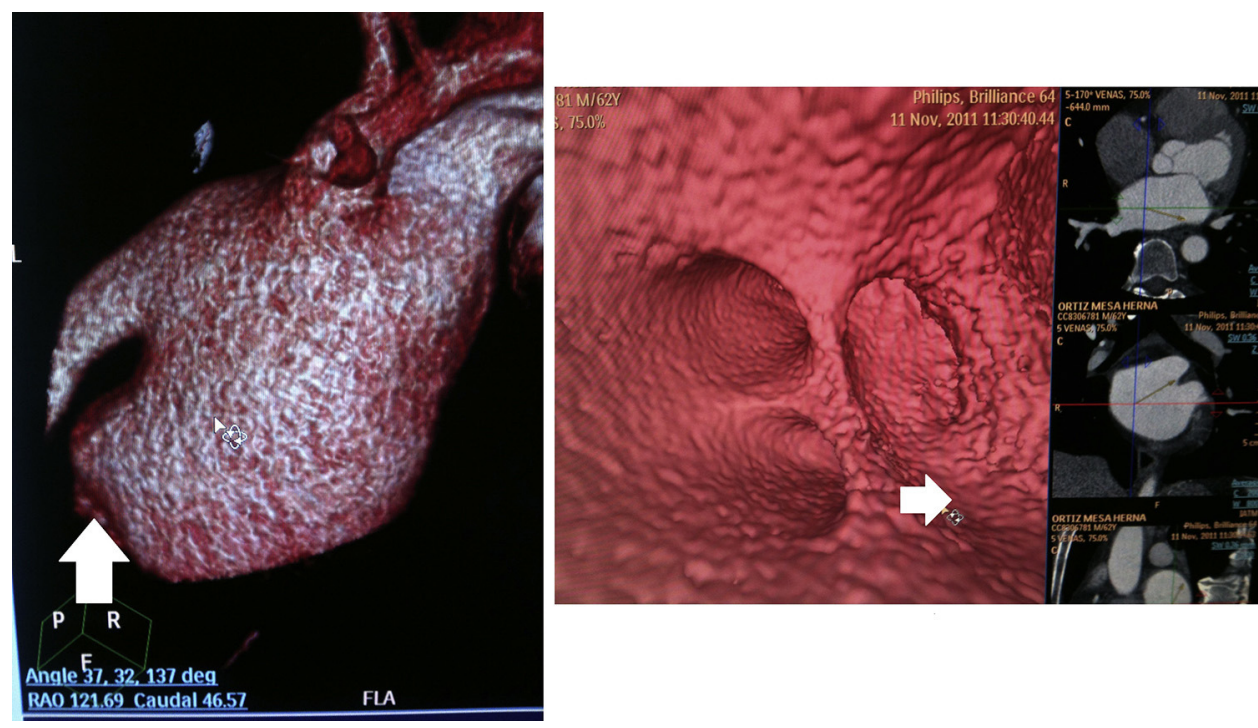

Figura 3 Evaginación del istmo mitral (flecha blanca), vista en reconstrucción volumétrica (izquierda) y en reconstrucción endocavitaria (derecha). 
Tabla 2 Características de las venas pulmonares

\begin{tabular}{|c|c|c|}
\hline Variable & \multicolumn{2}{|c|}{ Resultado } \\
\hline Venas accesorias & \multicolumn{2}{|c|}{$12(26 \%)$} \\
\hline Lado derecho & \multicolumn{2}{|c|}{$11(91,6 \%)$} \\
\hline Ostium común izquierdo & \multicolumn{2}{|c|}{$6(13 \%)$} \\
\hline Diámetro de venas pulmonares (AP, lat.) & Mayor & Menor \\
\hline VSD & $20 \pm 3,1 \mathrm{~mm}$ & $15,8 \pm 4,02 \mathrm{~mm}$ \\
\hline VID & $18,9 \pm 4,4 \mathrm{~mm}$ & $16,2 \pm 4,2 \mathrm{~mm}$ \\
\hline VSI & $21,9 \pm 5,5 \mathrm{~mm}$ & $14,6 \pm 4,3 \mathrm{~mm}$ \\
\hline VII & $17,8 \pm 2,9 \mathrm{~mm}$ & $11,6 \pm 2,8 \mathrm{~mm}$ \\
\hline \multicolumn{3}{|l|}{ Distancia a la primera bifurcación } \\
\hline VSD & $12,3 \pm 7,2 \mathrm{~mm}$ & \\
\hline VID & $8,7 \pm 5,3 \mathrm{~mm}$ & \\
\hline VSI & $22,7 \pm 10,4 \mathrm{~mm}$ & \\
\hline VII & $15,4 \pm 5,1 \mathrm{~mm}$ & \\
\hline
\end{tabular}

VID: vena inferior derecha; VII: vena inferior izquierda; VSD: vena superior derecha; VSI: vena superior izquierda.

no se halló tejido interpuesto entre el esófago y la vena pulmonar.

\section{Discusión}

Con el interés creciente en las intervenciones percutáneas para el manejo de la fibrilación auricular (la exclusión percutánea de la OAl o mediante el uso de dispositivo LARIAT $^{\circledR}$ y el aislamiento eléctrico de las venas pulmonares), cobra cada vez mayor relevancia el conocimiento preciso y detallado de la anatomía de la aurícula izquierda. Técnicas imaginológicas como la tomografía computarizada, la resonancia magnética o la ecocardiografía transesofágica (y de manera más reciente, la intracardíaca), han permitido obtener mayor información sobre la morfología de la aurícula izquierda. No obstante, se han descrito diferencias en el tamaño y la morfología de las aurículas entre poblaciones de distintas etnias, y aun en pacientes de la misma etnia obesos en relación con los no obesos (diferencias descritas desde la niñez), que impiden la extrapolación de los resultados obtenidos, de una población a otra ${ }^{17-19}$.

\section{Características morfológicas de la aurícula izquierda}

A diferencia del estudio de Koplay et al. ${ }^{15}$, en donde se encontró con mayor frecuencia el apéndice auricular tipo 2 b $(48 \%)$, en nuestro estudio la morfología más común fue la tipo 2 a (14 pacientes, 30,4\%). Sin embargo, se hallaron 2 orejuelas que no se ajustaron a la morfología descrita por ese grupo, el cual a su vez había descrito morfologías distintas a las establecidas por estudios previos ${ }^{20}$. Esto resalta las amplias variaciones existentes en la morfología del apéndice auricular y probablemente hace poco práctico el uso de las distintas clasificaciones que han sido propuestas.

Nuestros hallazgos tienen implicaciones importantes en el uso de dispositivos de cierre de orejuela, debido a que el empleo de estos depende en gran parte del diámetro del ostium de la orejuela (el dimensionamiento correcto de los dispositivos disminuye el riesgo de embolia del dispositivo) y en el caso del LARIAT $^{\circledR}$ de la orientación de la orejuela.
En nuestra serie, 11 pacientes (24\%) tuvieron un diámetro mayor que superaba los $28,5 \mathrm{~mm}$, punto de corte para el uso del dispositivo Cardiac plug ${ }^{\circledR}$ y $9(19,5 \%)$ tuvieron un diámetro mayor que superaba los $30 \mathrm{~mm}$, punto de corte para el dispositivo Watchman ${ }^{\circledR}$. Ningún paciente tuvo un diámetro que superara los $40 \mathrm{~mm}$ (diámetro máximo para el uso del LARIAT ${ }^{\circledR}$ ); no obstante en 5 casos $(11 \%)$ la ubicación superior y posterior de la punta del apéndice auricular impediría el uso de este dispositivo. Comparado con otras series, el diámetro del ostium de la orejuela fue similar a lo descrito previamente ${ }^{20}$; en este aspecto también hay grandes variaciones con otras descripciones de diámetros mucho menores a los encontrados en nuestro estudio ${ }^{21}$.

Conviene resaltar que se encontró evaginación del istmo mitral en el $35 \%$ de los casos, cifra mayor al $20 \%$ hallado en el estudio de Yokokawa, en el que la presencia de una evaginación se asoció con mayor probabilidad de requerir ablación a nivel del seno coronario para lograr la ablación del istmo $^{16}$. En quienes requieren ablación del seno coronario, la tasa de falla es mayor, particularmente si se encuentra una interposición de la arteria circunfleja y la pared auricular.

\section{Características de las venas pulmonares}

La frecuencia con la cual se encontraron venas pulmonares accesorias en nuestro estudio fue superior a la reportada en otras series (26 vs. 10-17\% respectivamente), la mayoría de ellas derechas ${ }^{11,22}$. El número de pacientes con ostium común (presente en el $26 \%$ de nuestros pacientes y encontrándose únicamente en el lado izquierdo en nuestra serie) y la distancia a la primera bifurcación (mayor en la vena superior izquierda y menor en la vena inferior derecha) fue similar a lo descrito en la literatura ${ }^{23,24}$. Estos hallazgos en las venas pulmonares concuerdan con el desarrollo embriológico de las mismas: las del lado derecho se desarrollan de manera más temprana que las del lado izquierdo, lo cual le permite a la aurícula expandirse hacia las venas pulmonares englobándolas de manera progresiva (las venas internalizadas se convierten progresivamente en la pared de la aurícula izquierda), lo que favorece el drenaje de cada una de ellas de manera independiente ${ }^{25}$. 
El diámetro de los ostium de las venas pulmonares en nuestro estudio fue superior a los diámetros encontrados en otras series ${ }^{26,27}$, lo cual tal vez esté relacionado con el punto establecido como ostium pulmonar, que en muchas series no ha sido detallado claramente o en los que se ha utilizado la deflexión del pericardio para establecer el ostium (distal al sitio de inserción de la vena en la aurícula en la mayoría de los casos). Lo mismo sucede con la distancia a la primera bifurcación (definida como el tronco de la vena pulmonar, el cual es mayor en las venas superiores que en las inferiores), cuya longitud varía de acuerdo con la serie estudiada y apoyaría esta teoría ${ }^{28}$.

El conocimiento de la anatomía de las venas pulmonares y la determinación precisa del ostium de la vena es fundamental para evitar estenosis postablación, que se estima ocurre en el $3 \%$ de las ablaciones realizadas dentro de la vena pulmonar (genera una disminución hasta del 68\% del diámetro de la vena), y de manera menos frecuente cuando se hace ablación ostial o antral (pese a ello se evidencia una disminución de aproximadamente un $8 \%$ del diámetro del ostium, la mayoría de ellos asintomáticos) ${ }^{28}$.

\section{Relación con el esófago}

Si bien no está establecida la incidencia real del daño esofágico asociado al proceso de ablación, aproximadamente el $47 \%$ de los pacientes sufre cambios en la mucosa, el $18 \%$ úlceras y cambios necróticos, y el 0,03 al $0,5 \%$ fístulas aurículo-esofágicas, temidas por su alta tasa de mortalidad $^{29-31}$. Se han descrito varios factores de riesgo asociados con mayor probabilidad de lesión esofágica, entre los que se encuentran aquellos relacionados con el aumento del paso de energía desde la aurícula izquierda al esófago, como el uso de altos niveles de energía o durante tiempo prolongado, el grosor de la pared auricular y la presencia y grosor del tejido graso entre la aurícula y el esófago respecto a la vena pulmonar. En nuestra serie, el $60 \%$ de los pacientes tuvieron contacto entre una o más venas pulmonares y el esófago, con grasa interpuesta tan solo en un $40 \%$ de las venas (mucho menor a lo reportado por Lemola et al.) ${ }^{32}$. Llama la atención que en nuestro estudio se observó mayor contacto de las venas pulmonares izquierdas con el esófago, fenómeno hallado solo por algunos autores y que podría explicarse por la motilidad del esófago, incluso durante el procedimiento de ablación ${ }^{33-35}$. Este hecho podría tener implicaciones prácticas importantes, ya que si bien el uso de anestesia general puede llegar a disminuir los movimientos del esófago, realizar una ablación agresiva en la pared posterior de la aurícula (independiente de si se hace sobre las venas pulmonares derechas o izquierdas), puede ser más riesgoso debido a las complicaciones previamente establecidas. En vista de la mortalidad asociada a la perforación esofágica, estimada en un $50 \%$, y a la tasa baja de perforaciones vistas en la práctica clínica, es probable que existan otros determinantes más relevantes.

\section{Limitaciones del estudio}

Los resultados no permiten establecer las características anatómicas de las aurículas de pacientes sanos e igualmente no son aplicables para pacientes con fibrilación auricular permanente, en quienes se han descrito diferencias anatómicas respecto a sujetos sanos e incluso con fibrilación auricular paroxística ${ }^{36}$. Pese a que varios estudios han encontrado mayor riesgo de recurrencias y complicaciones asociadas a ciertas características anatómicas, aún no se define con claridad el rol que desempeñan las variaciones anatómicas en la génesis y el mantenimiento de la fibrilación auricular. Adicionalmente, la definición de ostium de vena pulmonar fue distinta a la acepción más usual (según esta el ostium es el sitio donde se produce la reflexión del pericardio $)^{37}$. La razón para esta modificación tiene que ver con el aspecto práctico de la ablación; dado que por lo general se utilizan dispositivos de navegación tridimensional y fluoroscopia, durante el procedimiento no es posible determinar el sitio de reflexión del pericardio y la definición de aurícula, ostium y vena pulmonar en la mayoría de los casos se hace por las visiones endoscópicas que ofrecen estos dispositivos. Por esta razón, consideramos que la aproximación radiológica no es práctica para el cardiólogo intervencionista y usamos un método distinto, lo cual puede explicar algunas diferencias en los resultados obtenidos.

Otra limitación del estudio está relacionada con la clasificación morfológica de la orejuela izquierda empleada. Varios estudios han utilizado un esquema netamente morfológico que busca, mediante términos sencillos («cactus», «coliflor», «ala de pollo», «veleta»), describir el aspecto físico de la orejuela. Si bien esta clasificación tiene mayor difusión y hay estudios que demuestran como algunas de estas formas se relacionan con mayor riesgo embólico ${ }^{38-40}$, no introduce un componente fundamental para la intervención de la orejuela con algunos métodos novedosos, que por el contrario sí se logra con la clasificación que se usó en el estudio.

Por último, la medición de volúmenes y diámetros varía de manera considerable entre los distintos métodos imaginológicos (ecografía, tomografía computarizada, resonancia magnética); incluso entre mediciones efectuadas con el mismo método diagnóstico, puede haber variaciones significativas de acuerdo con el momento en el ciclo cardíaco en el cual se obtienen las imágenes. Por lo tanto, los hallazgos del estudio no pueden ser aplicados a las imágenes obtenidas con otros métodos diagnósticos.

\section{Conclusión}

En el estudio pudieron establecerse características morfológicas de la aurícula izquierda, las venas pulmonares y su relación con estructuras circundantes que son importantes, antes y durante la realización de procedimientos para el manejo de la fibrilación auricular (ablación de venas pulmonares y/o cierre de orejuela), siendo un punto de referencia con datos locales que se adaptan a nuestra población. Adicionalmente, puede servir como punto de partida para estudios posteriores que busquen determinar si hay factores predictores de fallos y complicaciones durante los procedimientos o recurrencia de la arritmia en el caso de ablación de venas pulmonares, lo que tendría un marcado impacto en la práctica de nuestros electrofisiólogos intervencionistas. En pacientes sometidos a procedimientos de intervención percutánea para fibrilación auricular (ablación de venas pulmonares y/o cierre de la orejuela), es fundamental el 
conocimiento de la anatomía previa al procedimiento, con el objetivo de mejorar la tasa de éxito y disminuir el riesgo de complicaciones. Adicionalmente, permite establecer una estrategia terapéutica específica para cada paciente.

\section{Responsabilidades éticas}

Protección de personas y animales. Los autores declaran que para esta investigación no se han realizado experimentos en seres humanos ni en animales.

Confidencialidad de los datos. Los autores declaran que en este artículo no aparecen datos de pacientes.

Derecho a la privacidad y consentimiento informado. Los autores declaran que en este artículo no aparecen datos de pacientes.

\section{Conflicto de intereses}

Los autores declaran no tener ningún conflicto de intereses.

\section{Agradecimientos}

A la Dra. Liliana Patricia Montoya Vélez, Coordinadora de Investigaciones de la Facultad de Medicina, Universidad CES.

\section{Bibliografía}

1. Wolf PA, Dawber TR, Thomas HE Jr, Kannel WB. Epidemiologic assessment of chronic atrial fibrillation and risk of stroke: the Framingham study. Neurology. 1978;28:973-7.

2. Feinberg WM, Blackshear JL, Laupacis A, Kronmal R, Hart RG. Prevalence age distribution, and gender of patients with atrial fibrillation. Analysis and implications. Arch Intern Med. 1995;155:469-73.

3. Haissaguerre M, Jais P, Shah DC, Takahashi A, Hocini M, Quiniou $G$, et al. Spontaneous initiation of atrial fibrillation by ectopic beats originating in the pulmonary veins. N Engl J Med. 1998;339:659-66.

4. Holmes DR, Reddy VY, Turi ZG, Doshi SK, Sievert H, Buchbinder $M$, et al. Percutaneous closure of the left atrial appendage versus warfarin therapy for prevention of stroke in patients with atrial fibrillation: a randomised non-inferiority trial. Lancet. 2009;374:534-42.

5. Aberg H. Atrial fibrillation. I. A study of atrial thrombosis and systemic embolism in a necropsy material. Acta Med Scandinavica. 1969;185:373-9.

6. Blackshear JL, Odell JA. Appendage obliteration to reduce stroke in cardiac surgical patients with atrial fibrillation. Ann Thorac Surg. 1996;61:755-9.

7. Stoddard MF, Dawkins PR, Prince CR, Ammash NM. Left atrial appendage thrombus is not uncommon in patients with acute atrial fibrillation and a recent embolic event: a transesophageal echocardiographic study. J Am Coll Cardiol. 1995;25:452-9.

8. Kato R, Lickfett L, Meininger G, Dickfeld T, Wu R, Juang G, et al. Pulmonary vein anatomy in patients undergoing catheter ablation of atrial fibrillation: lessons learned by use of magnetic resonance imaging. Circulation. 2003;107:2004-10.

9. Wang Y, Di Biase L, Horton RP, Nguyen T, Morhanty P, Natale A. Left atrial appendage studied by computed tomography to help planning for appendage closure device placement. J Cardiovasc Electrophysiol. 2010;21:973-82.
10. Walker DT, Humphries JA, Phillips KP. Anatomical analysis of the left atrial appendage using segmented, three-dimensional cardiac CT: a comparison of patients with paroxysmal and persistent forms of atrial fibrillation. J Interv Card Electrophysiol. 2012;34:173-9.

11. Wozniak-Skowerska I, Skowerski M, Wnuk-Wojnar A, Hoffmann A, Nowak S, Gola A, et al. Comparison of pulmonary veins anatomy in patients with and without atrial fibrillation: analysis by multislice tomography. Int J Cardiol. 2011;146:181-5.

12. Bittner A, Monnig G, Vagt AJ, Zellerhoff S, Wasmer K, Kobe $J$, et al. Pulmonary vein variants predispose to atrial fibrillation: a case-control study using multislice contrast-enhanced computed tomographyJT Europace. 2011;13:1394-400.

13. Monteiro MM, Saraiva C, Castelo Branco J, Cavaco D, Adragao P. Characterization of pulmonary vein morphology using multidetector row CT study prior to radiofrequency ablation for atrial fibrillation. Rev Port Cardiol. 2009;28:545-59.

14. Jang SW, Kwon BJ, Choi MS, Kim DB, Shin WS, Cho EJ, et al. Computed tomographic analysis of the esophagus, left atrium, and pulmonary veins: implications for catheter ablation of atrial fibrillation. J Interv Card Electrophysiol. 2011;32:1-6.

15. Koplay M, Erol C, Paksoy Y, Kivrak AS, Ozbek S. An investigation of the anatomical variations of left atrial appendage by multidetector computed tomographic coronary angiography. Eur J Radiol. 2012;81:1575-80.

16. Yokokawa M, Sundaram B >->, Garg A, Stojanovska J, Oral $\mathrm{H}$, Morady $\mathrm{F}$, et al. Impact of mitral isthmus anatomy on the likelihood of achieving linear block in patients undergoing catheter ablation of persistent atrial fibrillation. Heart Rhythm. 2011;8:1404-10.

17. Marcus GM, Olgin JE, Whooley M, Vittinghoff E, Stone KL, Mehra $\mathrm{R}$, et al. Racial differences in atrial fibrillation prevalence and left atrial size. Am J Med. 2010;123:375 e1-7e.

18. Dencker M, Thorsson O, Karlsson MK, Linden C, Andersen LB, Wollmer P. Body fat, abdominal fat, and body fat distribution is related to left atrial diameter in young children. Obesity (Silver Spring, Md). 2012;20:1104-8.

19. Kasper EK, Hruban RH, Baughman KL. Cardiomyopathy of obesity: a clinicopathologic evaluation of 43 obese patients with heart failure. Am J Cardiol. 1992;70:921-4.

20. Lacomis JM, Goitein O, Deible C, Moran PL, Mamone G, Madan $S$, et al. Dynamic multidimensional imaging of the human left atrial appendage. Europace: European pacing, arrhythmias, and cardiac electrophysiology. Europace. 2007;9:1134-40.

21. Ho SY, Cabrera JA, Sanchez-Quintana D. Left atrial anatomy revisited. Circ Arrhythm Electrophysiol. 2012;5:220-8.

22. Thorning C, Hamady M, Liaw JV, Juli C, Lim PB, Dhawan R, et al. CT evaluation of pulmonary venous anatomy variation in patients undergoing catheter ablation for atrial fibrillation. Clinical imaging. 2011;35:1-9.

23. Monteiro MM, Saraiva C, Castelo Branco J, Cavado D, Adragao P. Characterization of pulmonary vein morphology using multidetector row CT study prior to radiofrequency ablation for atrial fibrillation. Portuguese J Cardiol. 2009;28:545-59.

24. Cabrera JA, Sanchez-Quintana D. Cardiac anatomy: what the electrophysiologist needs to know. Heart (British Cardiac Society). 2013;99:417-31.

25. Stojanovska J, Cronin P. Computed tomography imaging of left atrium and pulmonary veins for radiofrequency ablation of atrial fibrillation. Semi Roentgenol. 2008;43:154-66.

26. Cronin P, Kelly AM, Desjardins B, Patel S, Gross BH, Kazerooni EA, et al. Normative analysis of pulmonary vein drainage patterns on multidetector CT with measurements of pulmonary vein ostial diameter and distance to first bifurcation. Acad Radiol. 2007;14:178-88.

27. Kim YH, Marom EM, Herndon JE, McAdams HP. Pulmonary vein diameter, cross-sectional area, and shape: CT analysis. Radiology. 2005;235:43-9. Discussion 9-50. 
28. Scharf C, Sneider M, Case I, Chugh A, Lai SW, Pelosi F Jr, et al. Anatomy of the pulmonary veins in patients with atrial fibrillation and effects of segmental ostial ablation analyzed by computed tomography. J Cardiovasc Electrophysiol. 2003;14:150-5.

29. Cummings JE, Schweikert RA, Saliba WI, Burkhardt JD, Kilikaslan F, Saad E, et al. Brief communication: atrial-esophageal fistulas after radiofrequency ablation. Ann Int Med. 2006;144: $572-4$.

30. Ghia KK, Chugh A, Good E, Pelosi F, Jongnarangsin K, Bogun F, et al. A nationwide survey on the prevalence of atrioesophageal fistula after left atrial radiofrequency catheter ablation. pacing Interv Card Electrophysiol. 2009;24:33-6.

31. Schmidt M, Nolker G, Marschang H, Gutleben KJ, Schibgilla V, Rittger $\mathrm{H}$, et al. Incidence of oesophageal wall injury post-pulmonary vein antrum isolation for treatment of patients with atrial fibrillation. Europace. 2008;10: 205-9.

32. Lemola K, Sneider M, Desjardins B, Case I, Han J, Good E, et al. Computed tomographic analysis of the anatomy of the left atrium and the esophagus: implications for left atrial catheter ablation. Circulation. 2004;110:3655-60.

33. Bahnson TD. Strategies to minimize the risk of esophageal injury during catheter ablation for atrial fibrillation. PACE. 2009;32:248-60.
34. Jang SW, Kwon BJ, Choi MS, Kim DB, Shin WS, Cho EJ, et al. Computed tomographic analysis of the esophagus, left atrium, and pulmonary veins: implications for catheter ablation of atrial fibrillation. J Interv Electrophysiol. 2011;32:1-6.

35. Han J, Good E, Morady F, Oral H. Images in cardiovascular medicine. Esophageal migration during left atrial catheter ablation for atrial fibrillation. Circulation. 2004;110:e528.

36. Imada M, Funabashi N, Asano M, Uehara M, Ueda M, Komuro I. Anatomical remodeling of left atria in subjects with chronic and paroxysmal atrial fibrillation evaluated by multislice computed tomography. Int J Cardiol. 2007;119:384-8.

37. Casella A. An atlas of radioscopic catheter placement for the electrophysiologist. London: Springer; 2008.

38. Di Biase L, Santangeli P, Anselmino M, Mohanthy P, Salvetti I, Gili $S$, et al. Does the left atrial appendage morphology correlate with the risk of stroke in patients with atrial fibrillation? Results from a multicenter study. J Am Coll Cardiology. 2012;60:531-8.

39. Anselmino M, Scaglione M, Di Biase L, Gili S, Santangeli P, Corsinovi L, et al. Left atrial appendage morphology and silent cerebral ischemia in patients with atrial fibrillation. Heart Rhythm. 2014;11:2-7.

40. Kimura T, Takatsuki S, Inagawa K, Katsumata Y, Nishiyama T, Nishiyama N, et al. Anatomical characteristics of the left atrial appendage in cardiogenic stroke with low $\mathrm{CHADS}_{2}$ scores. Heart Rhythm. 2013;10:921-5. 